\title{
Blood platelet behaviour during infusion of an Intralipid-based intravenous feeding mixture
}

\author{
W. R. BURNHAM \\ M.B., M.R.C.P. \\ *S. R. CocKBILL \\ B.Sc.
}

*S. HePTINSTALl

B.Sc., Ph.D.

S. HARRISON

B.Med. Sci.

\section{Department of Therapeutics, City Hospital, Nottingham and *Department of Medicine, University Hospital, Queens Medical Centre, Nottingham}

\begin{abstract}
Summary
Blood platelet behaviour was studied in six patients during infusion of an Intralipid-based intravenous feeding mixture. Lower concentrations of sodium arachidonate were required to induce platelets to aggregate and to undergo a release reaction during intravenous feeding than were required before or after intravenous feeding. This change was not due to a change in serum albumin level. The platelet behaviour that was induced by adenosine diphosphate or by adrenaline remained unchanged during intravenous feeding.
\end{abstract}

\section{Introduction}

The first fat emulsions to be used extensively for intravenous feeding were found to be toxic. Among the various side effects were bleeding phenomena that were associated with coagulation defects, and thrombocytopenia. Although modern fat emulsions such as Intralipid are considered to be safe, there has been one report of thrombocytopenia after prolonged use (Lipson, 1974). Fat emulsions may lead to thrombocytopenia through an effect on platelet function and Atik (1976) has suggested that evaluation of platelet function in conjunction with current and future fat emulsions for human use is to be recommended.

Intravenous nutrition can be simplified if Intralipid is mixed with other nutrients in a single 3litre bag before infusion (Burnham et al., 1981). The purpose of the present study was to compare blood platelet behaviour before, during and after infusion of the mixture that is currently being used.

\section{Materials and methods}

\section{Patients}

Six patients who required intravenous nutrition were studied. Five of the patients had inflammatory bowel disease requiring surgery; the other had a diverticular abscess and intestinal obstruction. The ages of the patients ranged from 27 to 67 years (mean 49 years). The duration of intravenous $\vec{z}$ feeding, together with the points at which surgery was performed and the points at which blood $\stackrel{\circ}{S}$ samples for the platelet studies were withdrawn, $\vec{\theta}$ are summarized in Fig. 1.

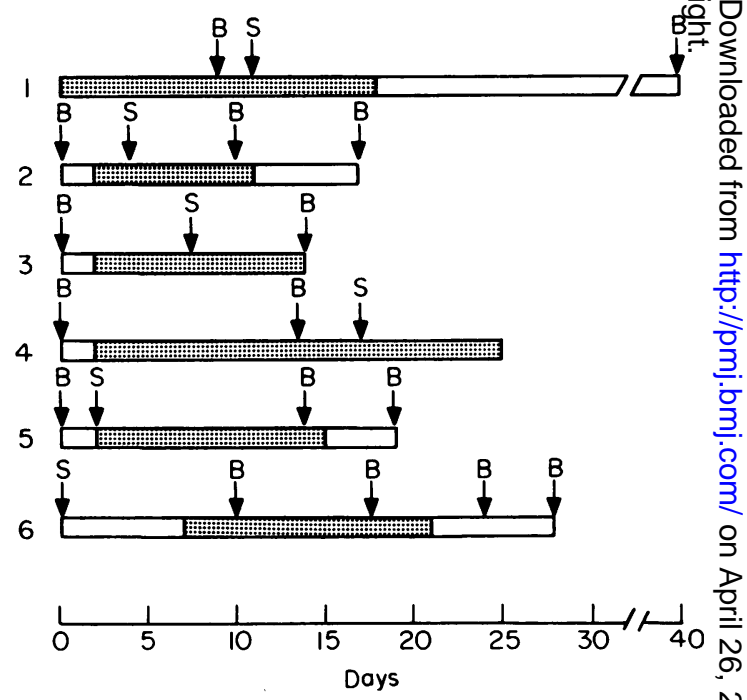

FIG. 1. The points at which surgery was performed (S) and at which blood samples were taken for the platelet studies $\mathrm{W}$ (B) for each of the six patients. The shaded areas indicated $\sigma$ the period for which the Intralipid-feeding mixture was infused.

Infusion mixture

The basic composition of the mixture that was used is as follows: Intralipid ${ }^{\circledR}(20 \%, 1$ litre $)$, Vamin $\underset{\mathbb{D}}{ }$ 
glucose $^{\circledR}$ (1.5 litre), dextrose $(10 \%, 0.5$ litre $)$, potassium chloride $(54 \mathrm{mmol})$, potassium dihydrogen phosphate $(30 \mathrm{mmol})$, sodium chloride $(30 \%$, $52 \mathrm{mmol}$ ), Addamel $\mathrm{R}$ (a calcium, magnesium and trace element solution, $10 \mathrm{ml}$ ), Vitlipid ${ }^{\circledR}$ (fat soluble vitamins, 1 vial), Solivito ${ }^{\circledR}$ (water soluble vitamins, 1 vial). For three of the six patients (nos 1, 3, and 5), heparin (2000 i.u.) was added to the bag. When mixed under laminar air flow conditions and then infused over a 24 -hr period, it provided $1.2 \times 10^{7}$ non-protein $\mathrm{J}, 14 \mathrm{~g}$ of nitrogen, $126 \mathrm{mmol}$ of sodium, $114 \mathrm{mmol}$ of potassium, $30 \mathrm{mmol}$ of phosphate, $8.75 \mathrm{mmol}$ of calcium, $3.75 \mathrm{mmol}$ of magnesium, trace elements and vitamins.

\section{Platelet behaviour}

Blood samples $(40 \mathrm{ml})$ were collected into four tubes $(10 \mathrm{ml}$ capacity) that each contained 100 units of heparin (Weddel Pharmaceuticals Ltd) and 0.3 $\mu \mathrm{Ci}$ of ${ }^{14} \mathrm{C}$-serotonin (Radiochemical Centre, Amersham; specific activity $58 \mathrm{mCi} / \mathrm{mmol}$ ). The heparin served to anticoagulate the blood; the ${ }^{14} \mathrm{C}$-serotonin served to label the platelets in the sample before studies of the platelet release reaction. Platelet-rich plasma (PRP) and platelet-poor plasma (PPP) were prepared from the blood by differential centrifugation and the number of platelets in the preparations was determined using a Coulter counter. The PPP was used to dilute the PRP to a platelet count of $300 \times 10^{9} / 1$.

It was not possible to use the aggregometer to assess the effect of Intralipid on platelet behaviour because the presence of lipid particles in some of the preparations of PRP masked the changes in light absorbance that occurred. Instead, the authors measured the extent to which the platelets could be induced to undergo the platelet release reaction in response to various stimuli. The extents to which platelets aggregate and undergo the platelet release reaction are related (Heptinstall and Mulley, 1977). The stimuli that were used were adenosine diphosphate (ADP, final concentration $10 \mu \mathrm{mol} / \mathrm{l}$ ), adrenaline (final concentration $10 \mu \mathrm{mol} / \mathrm{l}$ ) and sodium arachidonate (NaAA, obtained as arachidonic acid from the Sigma Chemical Co. and converted into the sodium salt; final concentrations $0 \cdot 1,0 \cdot 2$, $0 \cdot 4,0 \cdot 6,0.8$ and $1.0 \mathrm{mmol} / \mathrm{l})$. In each case, the extent of the platelet release reaction was determined by measuring the amount of ${ }^{14} \mathrm{C}$-serotonin that was released from the cells. This was expressed as a percentage of the amount of ${ }^{14} \mathrm{C}$-serotonin that was taken up by the platelets during the labelling procedure. The techniques that were used and the procedures that were adopted have already been described (Cockbill, Heptinstall and Taylor, 1979).

Serum albumin and triglyceride concentrations were determined using standard procedures at various stages before, during and after infusion of the intravenous feeding mixture. An antifactor $\mathrm{Xa}$ assay was used to determine serum heparin.

\section{Results}

ADP and adrenaline always induced platelet aggregation when added to PRP. The amount of ${ }^{14} \mathrm{C}$-serotonin that was released as the platelets

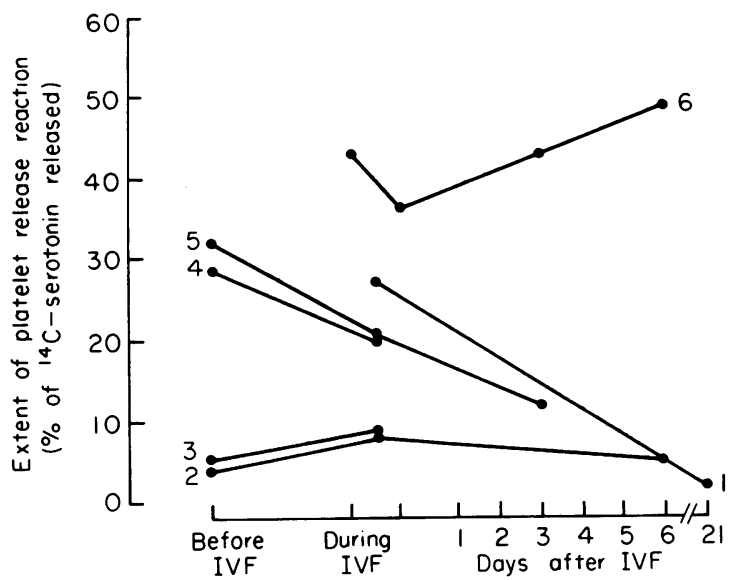

FIG. 2. The extent of the ADP-induced platelet release reaction during intravenous feeding (IVF).

aggregated are shown in Fig. 2 and 3. For two patients (nos 2 and 3 ) the amount of ${ }^{14} \mathrm{C}$-serotonin that was released in response to ADP or adrenaline was always less than $10 \%$ of the amount of ${ }^{14} \mathrm{C}$ serotonin that had been taken up by the platelets during the labelling procedure. For one patient (no. 6) it was always $>35 \%$. For two patients (nos 4 and 5) intermediate levels of ${ }^{14} \mathrm{C}$-serotonin were released in response to both ADP and adrenaline. Only for one patient (no. 1) was there a substantial change in the amount of ${ }^{14} \mathrm{C}$-serotonin released during intravenous feeding.

There is a threshold level of sodium arachidonate below which the material will not induce platelet aggregation or any release reaction in PRP. Above this threshold level aggregation occurs and the amount of ${ }^{14} \mathrm{C}$-serotonin that is released from the platelets as the platelets aggregate is always $>40 \%$ of the amount taken up by the platelets during the labelling procedure. Figure 4 shows that the threshold concentration of arachidonate that was required for aggregation and the release reaction to occur, changed during intravenous feeding. In all six patients the amount of arachidonate that was required to induce aggregation and the release reaction was reduced. In one (no. 6) the threshold level fell during intravenous feeding and then fell 


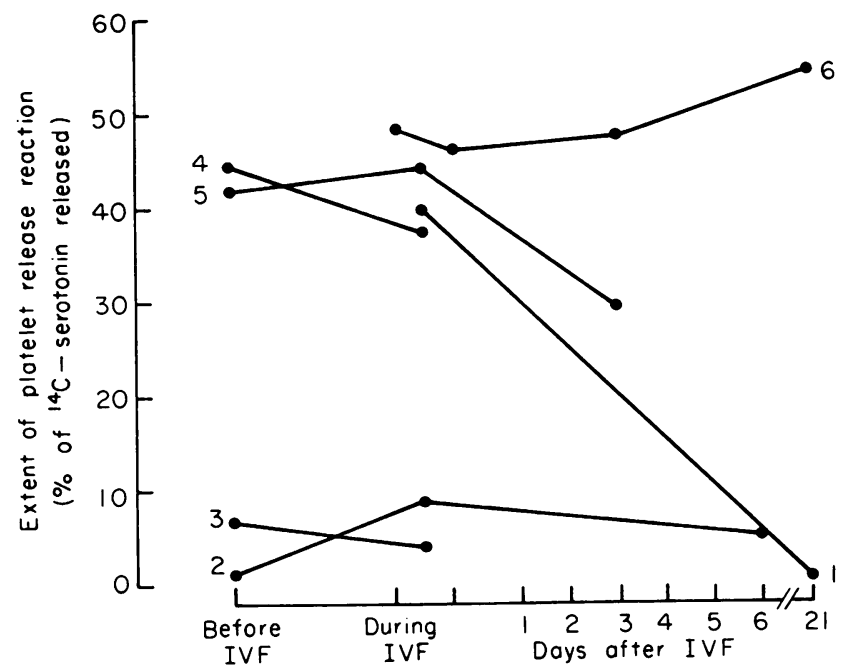

FIG. 3. The extent of the adrenaline-induced platelet release reaction during intravenous feeding (IVF).

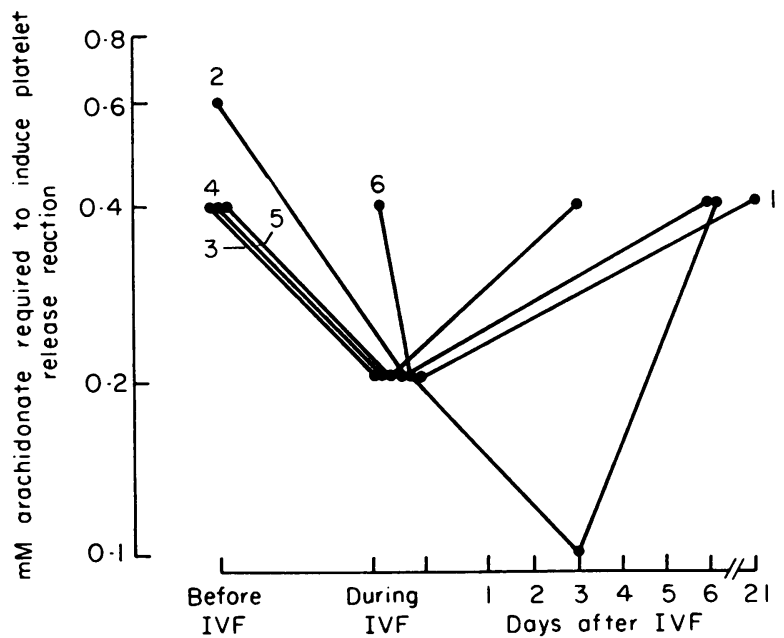

Fig. 4. The concentrations of arachidonate $(\mathrm{mmol})$ that were required to induce a release reaction in platelets during intravenous feeding (IVF).

further three days after intravenous feeding had been terminated; 6 days after intravenous feeding had been terminated it had risen again. In another patient (no. 5) the threshold had returned to normal after only 3 days. The concentrations of albumin in serum from the patients varied during the procedures but changes did not correspond to the changes in arachidonate threshold. The variation in albumin levels was consistent in each case with the hydration of the patient and the severity of their illness.

No clinical evidence of a bleeding or thrombotic tendency was noted in any of the patients. Plasma heparin concentrations were always below 0.03 i.u./ml in those patients who received heparin in their feeds. Serum triglyceride concentrations during the feeding were determined in 5 of the 6 patientso They were always between 1.1 and $1.9 \mathrm{mmol} / 1$ (within the normal range) thus indicating that the infuse lipid was being metabolized at an adequate rate.

\section{Discussion}

Platelets from different individuals vary in the extent to which they release labelled serotonin when stimulated by the aggregating agents ADP and adrenaline (Heptinstall and Mulley, 1977). In this study, infusion of an Intralipid-based mixture did not, with one exception, significantly alter the extent of the platelet release reaction. The exception was in the case of platelets obtained from a patient with very severe Crohn's disease of the colon which failed to respond to high doses of intravenous prednisolone. The other patients studied were less ill and this may explain the difference in the results. Some of the authors' unpublished data suggest that severely injured patients may have a temporarily increased platelet reactivity; very severe large bowel Crohn's disease may be a parallel situation.

In all of the patients studied, the concentration of sodium arachidonate needed to induce platelets to aggregate and to release labelled serotonin was lower during infusion than before or after infusion. Such a change could result from activation of platelet cyclo-oxygenase, the enzyme responsible for converting arachidonate into the prostaglandins and thromboxanes that are responsible for the aggregation and release reaction. The apparent 
lack of effect of the infused Intralipid mixture on ADP- and adrenaline-induced platelet behaviour, however, tends to argue against cyclo-oxygenase activation as the explanation of this phenomenon since effects of ADP and adrenaline on platelets are also mediated by this enzyme.

It is possible that a reduction in the threshold level of arachidonate that was required to induce aggregation and release stems from changes that occur in the blood plasma itself during infusion of the Intralipid mixture. A reduced threshold might theoretically occur if the concentration of plasma proteins were reduced. However, any change in serum albumin that did occur, did not occur in parallel with the change in arachidonate threshold. Another possibility is that basal concentrations of circulating fatty acid were increased during the intravenous feeding. Certainly the normal triglyceride concentrations that were observed during intravenous feeding suggest that the lipid was being actively metabolized. Unfortunately, however, the free fatty acid levels were not measured.

If increased free fatty acid concentrations in the plasma were causing a decreased platelet release threshold to arachidonic acid in vitro, there are several ways in which this could have occurred. The mixing of the Intralipid and other nutrients may have caused release of free fatty acid. However, when platelet concentrates were treated with the mixture in vitro, no change in the release threshold to arachidonic acid was observed. Thus, lipolysis must have occurred in vivo only. The presence of heparin in some feeds might have released lipoprotein lipase into the plasma causing increased lipolysis; but, platelets from the three patients receiving heparin in the feed behaved in the same way as platelets from patients not receiving heparin. It is concluded that free fatty acid concentrations may have been elevated in the plasma of these patients owing to increased lipolysis in vivo; if so, low dose heparin infusion did not affect this.

No patient developed clinical evidence of bleeding or thrombotic tendency nor did the authors find any evidence that such problems might have developed owing to altered platelet behaviour. However, the change in threshold for arachidonic acid is disturbing. One of the fatty acids present in Intralipid is arachidonic acid itself. If the altered threshold to arachidonate is consequent to free fatty acids generated from Intralipid, it is theoretically possible that these free fatty acids could activate the platelets directly. Perhaps more rapid infusions than those used in this study would give rise to very high concentrations of free fatty acid, and should be avoided.

\section{References}

AtIK, M. (1976) Platelet dysfunction after intravenous infusion of fat emulsion. Acta chirurgica scandinavica, 466 (Suppl.), 68.

Burnham, W.R., Hansrani, P., KNOtT, C.F., CoOK, J., Davis, S.S. \& Langman, M.J.S. (1981) Simplified intravenous nutrition, using Intralipid-based mixture in a 3 litre bag. Gut, 22, 897.

Cockbill, S.R., Heptinstall, S. \& Taylor, P.M. (1979) A comparison of the abilities of acetylsalicylic acid, flurbiprofen and indomethacin to inhibit the release reaction and prostaglandin synthesis in human blood platelets. British Journal of Pharmacology, 67, 73.

Heptinstall, S. \& Mulley, G.P. (1977). Adenosine diphosphate-induced platelet aggregation and release reaction in heparinized platelet rich plasma and the influence of added citrate. British Journal of Haematology, 36,565 .

Lipson, A.H. (1974) Thrombocytopenia after Intralipid infusion in a neonate. Lancet, ii, 1462. 